\title{
Atomistic Structure Simulation of Silicon Nanocrystals Driven with Suboxide Penalty Energies
}

Article in Journal of Nanoscience and Nanotechnology · March 2008

DOI: 10.1166/jnn.2008.A117 · Source: PubMed

CITATIONS

2

3 authors, including:

-

Dundar Yilmaz

Pennsylvania State University

19 PUBLICATIONS 114 CITATIONS

SEE PROFILE
READS

10

Tcagin Cagin

Texas A\&M University

255 PUBLICATIONS 6,681 CITATIONS

SEE PROFILE

Some of the authors of this publication are also working on these related projects:

Project Carbon Nanotube Polymer Nanocomposite for Electromechanical System Applications View project

Project First - principles calculations on stability and mechanical properties of various ABO 3 and their alloys View project 


\title{
Atomistic Structure Simulation of Silicon Nanocrystals Driven with Suboxide Penalty Energies
}

\author{
D. E. Yılmaz ${ }^{1,2, *}$, C. Bulutay ${ }^{1,2}$, and T. Çagın ${ }^{3}$ \\ ${ }^{1}$ Department of Physics, Bilkent University, Ankara 06800, Turkey \\ ${ }^{2}$ National Nanotechnology Research Center, Bilkent University, Ankara 06800, Turkey \\ ${ }^{3}$ Artie McFerrin Department of Chemical Engineering, Jack E. Brown Engineering Building, Texas A\&M University, \\ 3122 TAMU, College Station, TX 77843-3122, USA
}

\begin{abstract}
The structural control of silicon nanocrystals embedded in amorphous oxide is currently an important technological problem. In this work, an approach is presented to simulate the structural behavior of silicon nanocrystals embedded in amorphous oxide matrix based on simple valence force fields as described by Keating-type potentials. After generating an amorphous silicon-rich-oxide, its evolution towards an embedded nanocrystal is driven by the oxygen diffusion process implemented in the form of a Metropolis algorithm based on the suboxide penalty energies. However, it is observed that such an approach cannot satisfactorily reproduce the shape of annealed nanocrystals. As a remedy, the asphericity and surface-to-volume minimization constraints are imposed. With the aid of such a multilevel approach, realistic-sized silicon nanocrystals can be simulated. Prediction for the nanocrystal size at a chosen oxygen molar fraction matches reasonably well with the experimental data when the interface region is also accounted. The necessity for additional shape constraints suggests the use of more involved force fields including long-range forces as well as accommodating different chemical environments such as the double bonds.
\end{abstract}

Keywords: Silicon Nanocrystals, Simulation, Structure.

\section{INTRODUCTION}

Silicon nanocrystals (NCs) embedded in amorphous silicon dioxide $\left(\mathrm{a}-\mathrm{SiO}_{2}\right)$ promise interesting applications such as nonvolatile memories, visible light emitting diodes and solar cells. Understanding the atomistic structure of this system will be invaluable in the development and optimization of such devices. An atomistic computer model for this system is the main subject of this work. Realistic computer simulation of such a system requires very large supercells containing at least several thousand atoms. This makes impossible to use ab initio methods with the current status of the algorithms and computer powers. Instead one should use a simpler model to describe the system. The seminal work for the generation of amorphous systems was proposed by Wooten, Winer and Weaire (WWW). ${ }^{1}$ They used Keating potential ${ }^{2}$ to describe the system where the total energy of system depends on the deviation of bond length and angles between adjacent bonds from the ideal crystal case. Starting with diamond crystal, applying random bond switches they were able to generate a realistic

${ }^{*}$ Author to whom correspondence should be addressed. model for 216-atom a-Si. Using WWW approach one can generate $\mathrm{a}-\mathrm{SiO}_{2}$ or a- $\mathrm{GeO}_{2}$ structures.

Based on ab initio calculations on small Si clusters representing nonstoichiometric systems Hamann showed that the energy of $\mathrm{Si}$ atoms varies with oxidation states. ${ }^{3}$ This variation is referred to as "suboxide penalty." In such a system the suboxide penalty favors silicon atoms bonding with either four oxygen atoms or four silicon atoms. Using this fact Burlakov et al. modeled the phase separation of nonstoichiometric amorphous silica. ${ }^{4}$ They mapped Metropolis Monte Carlo simulations to rate equations thereby extracting the suboxides densities. With a small modification the WWW method can also be applied to nonstochiometric systems. Tu et al. worked on the structure and energetics of $\mathrm{Si}_{-} \mathrm{SiO}_{2}$ interface by introducing additional term to Keating potential. ${ }^{5}$ This additional term was the suboxide penalty calculated by Hamann. ${ }^{3}$ The $\mathrm{Si}_{-} \mathrm{SiO}_{2}$ interface plays a crucial role in the physical properties of $\mathrm{Si} \mathrm{NCs}$ embedded in $\mathrm{a}-\mathrm{SiO}_{2}$. Recently, Hadjisavvas and Kelires used WWW method to study Si NCs embedded in a-SiO $2 .{ }^{6}$ They first generated $\mathrm{a}-\mathrm{SiO}_{2}$ system using WWW method. Next, within a determined radius, they removed all 
oxygen atoms to form a Si NC. Their major finding was the importance of $\mathrm{Si}-\mathrm{O}-\mathrm{Si}$ bridge bonds. ${ }^{6}$

In this work, we report a multilevel algorithm to simulate the atomistic structure of $\mathrm{Si} \mathrm{NC}$ embedded in a- $\mathrm{SiO}_{2}$. Our approach starts with the generation of $\mathrm{a}-\mathrm{SiO}_{2}$ with excess silicon so as to enable $\mathrm{Si}$ NC formation. This part is based on the standard WWW model. Next, with a crucial insight from Burlakov et al., ${ }^{4}$ the evolution of the silicon-rich-oxide towards an embedded NC is driven by the oxygen diffusion process, implemented in the form of a Metropolis algorithm based on the suboxide energies determined from $a b$ initio computations. ${ }^{3}$ This is followed by the shape constraints to attain a spherical geometry. The details of the approach and the results are contained in the following sections.

\section{COMPUTATIONAL APPROACH AND RESULTS}

\subsection{Generating Amorphous Silicon-Rich-Oxide}

As the first step, to generate amorphous silicon-rich-oxide we start with a 1000-atom diamond Si crystal. The energetics of the system is described by Keating-type twobody and three-body potentials. ${ }^{2}$ However, since the bonds are implicitly defined and updated during simulation, nonbonded atoms can approach very close to each other. To prevent this, we include an extra term to Keating potential, to penalize atoms which are non-bonded and closer than some critical distance which is a well known recipe. ${ }^{7}$ Using WWW random bond transpositions, we generate a-Si system with $13^{\circ} \mathrm{rms}$ bond angle deviation of $\mathrm{Si}-$ $\mathrm{Si}-\mathrm{Si}$ bonds and $0.3 \AA \mathrm{rms}$ bond length deviation of the $\mathrm{Si}-\mathrm{Si}$ bonds. In the WWW method after each bond transposition the system is relaxed using conjugate gradient minimization scheme to decide whether to accept or reject the step based on a Metropolis algorithm. The usual temperature factor enters the decision process. Since we start with an ideal crystal structure we need to erase the traces of the initial topology. This is achieved by randomizing the system by accepting all bond transpositions (i.e., temperature of system being set to infinity) till system reaches $23^{\circ} \mathrm{rms}$ bond angle deviation for the $\mathrm{Si}-\mathrm{Si}-\mathrm{Si}$ bonds. After this point we set the temperature of system to $k T=0.40 \mathrm{eV}$ and continue random bond transpositions. In this way energy of system decreases and it cools down to an amorphous state with a desired rms bond angle deviation. Radial distribution function is the main diagnostic tool to compare simulation results with experiment. In Figure 1 we present graph of radial distribution function versus distance from a chosen atom in a-Si. First peak resembles mean bond length of $\mathrm{Si}-\mathrm{Si}$ bonds while second peak's width resembles bond angle deviation of $\mathrm{Si}-\mathrm{Si}-$ $\mathrm{Si}$ bond angle. After preparing the 1000-atom a-Si system we generate amorphous silicon-rich-oxide (i.e., $\mathrm{SiO}_{x}$ with $x<2$ ) by randomly inserting required amount of oxygen

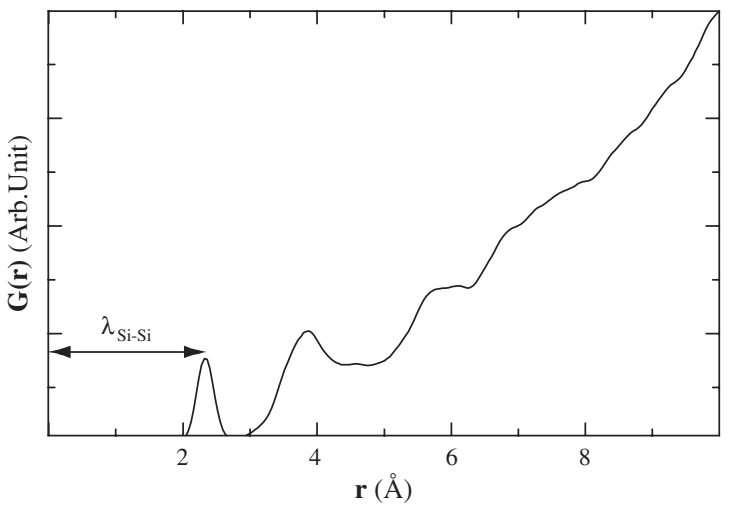

Fig. 1. Radial distribution function of amorphous Si. First peak resembles $\mathrm{Si}-\mathrm{Si}$ bond length. Second peak's width resembles bond angle deviation.

atoms between $\mathrm{Si}$ atoms. After the insertion of oxygen atoms we expand the simulation box to acquire the correct density. Next, we relax the system using conjugate gradient method.

\subsection{Evolution Towards Nanocrystals}

In our simple approach, the amorphous silicon-rich-oxide is driven towards nanocrystal formation by the diffusion of oxygen atoms, illustrated in Figure 2. In its implementation as a Monte Carlo (MC) step, first a silicon atom with oxidation state between 1 to 3 is randomly chosen, then one of oxygen neighbors of this silicon atom is transferred to the midpoint of $\mathrm{Si}-\mathrm{Si}$ bond of this silicon. Difference of the initial and final suboxide energies of system is used in the decision of the step. The temperature parameter for this step is $k T=0.15 \mathrm{eV}$. Since oxygen diffusion is much slower than the relaxation process we assume that system is fully relaxed during this step. This assumption accelerates evolution of system. After $10^{6} \mathrm{MC}$ diffusion steps which are carried out in less then two hours with a standard computer, the excess silicon atoms start forming nanoclusters.

A silicon suboxide, denoted by $\operatorname{Si}(i)$ is defined as a silicon atom bonded with $i$ oxygens. Since $\operatorname{Si}(1)$ and $\operatorname{Si}(2)$ atoms lie at the surface of $\mathrm{NC}$ while $\mathrm{Si}(3)$ atoms lie mainly in the oxide region, densities of the suboxides determine the shape of the NC. In other words, suboxide energies play a crucial role in the determination of the shape of the NC. In our simulation, the use of suboxide energies calculated by $a b$ initio methods ${ }^{3}$ results in a toroid-like shaped NCs (cf. Fig. 3). However TEM images show that Si NCs have spherical forms. ${ }^{8}$ This situation forced us to modify suboxide energies to end up with a sphere-like NC. To modify suboxide energies we make use of Burlakov's rate equation approach. ${ }^{4}$ First, we extract the suboxide densities of a spherical NC by preparing an "ideal" $\mathrm{NC}$ embedded in $\mathrm{a}-\mathrm{SiO}_{2}$ achieved by deleting all oxygen atoms in an a- $\mathrm{SiO}_{2}$ system within a given radius as in Ref. [6]. Then, 

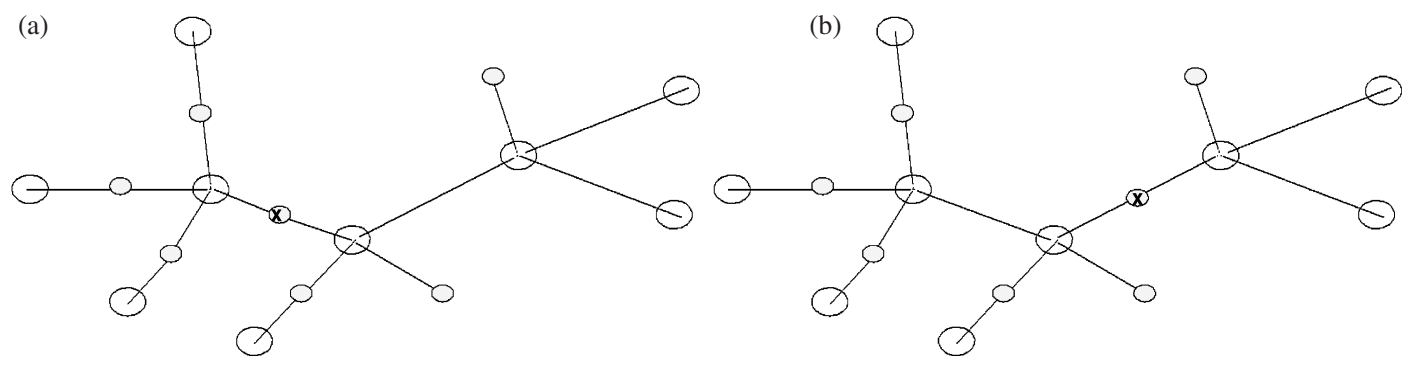

Fig. 2. Illustration of the oxygen diffusion steps: Black spheres represent silicon atoms, dashed spheres represents oxygen atoms. In (a) oxidation states of atom A and atom B are 4 and 1, respectively. After the oxygen diffusion (b) oxidation state of atom A is decreased to 3 while oxidation state of atom $\mathrm{B}$ is increased to 2 .

using Burlakov's rate equation we fit suboxide energies to achieve suboxide densities calculated from the "ideal" NC. Unfortunately, this atempt of modifying suboxide energies was not sufficient to force the system to form sphere-like $\mathrm{NC}$; instead it leads to a cylinder-like shape as shown in Figure 4.

To closely monitor the geometry of the NC, its quantitative assessment can be achieved by diagonalizing the shape tensor of the $\mathrm{NC}$ which is given by: ${ }^{9}$

$$
G_{i j}=\frac{1}{N} \sum_{n=1}^{N}\left(r_{i n}-R_{i}\right)\left(r_{j n}-R_{j}\right)
$$

here $N$ is the number of NC atoms, $R_{i}$ 's are the coordinates of the center-of-mass of the $\mathrm{NC}$ and $r_{i n}$ is the $i$ th coordinate of $n$ 'th NC atom. The ratio of three eigenvalues of shape tensor $g_{1}, g_{2}$, and $g_{3}$ (in descending order) determine the shape of the NC. Deviation from a perfect sphere (asphericity) is quantified by Gaspari and Rudnick ${ }^{10}$ as

$$
\delta=1-3 \frac{I_{2}}{I_{1}^{2}}
$$

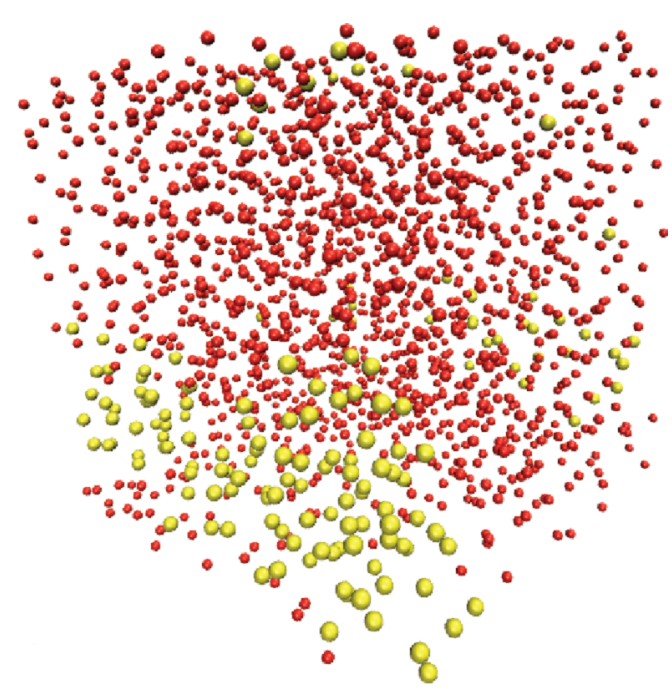

Fig. 3. (Color online) Snapshot of simulation after steady-state is reached, using $a b$ initio suboxide energies from Ref. [3]. Only oxygen atoms (dark colored) and zero-oxidation-state silicon atoms (bright colored) are shown. Periodic boundary conditions are imposed around the simulation box.
In this equation, $I_{1}=g_{1}+g_{2}+g_{3}$ and $I_{2}=g_{1} g_{2}+g_{2} g_{3}+$ $g_{1} g_{3}$ are the respective invariants of shape tensor. For a perfect sphere eigenvalues of the shape tensor are equal so that the asphericity parameter is equal to zero.

The asphericity parameter for $\mathrm{NC}$ formed using $a b$ initio suboxide energies (Fig. 3) is 0.40 while for the $\mathrm{NC}$ formed using suboxide energies extracted from "ideal" NC (Fig. 4) it becomes 0.23 . So these NCs deviate too much from a perfect sphere. Since surface tovolume ratio is minimum for a sphere we also calculate the ratio of number of surface atoms to number of $\mathrm{NC}$ atoms. This ratio was also much larger than the "ideal" NC case.

These observations reveal the importance of long-range forces lacking in these attempts. However, somewhat artificially, the inclusion of asphericity parameter and surfaceto-volume ratio can lead to better results. In our simulation with the modified suboxide energies, after the steady state is reached, we continue with MC steps but this time we include asphericity parameter and the surface-to-volume ratio to Metropolis decision. Inclusion of these parameters results in the formation of $\mathrm{NC}$ with asphericity parameter less then 0.001 and also $g_{1} / g_{2}$ falls in the range $1.0-1.1$ and $g_{1} / g_{3}$ falls in the range $1.1-1.3$. This means that inclusion

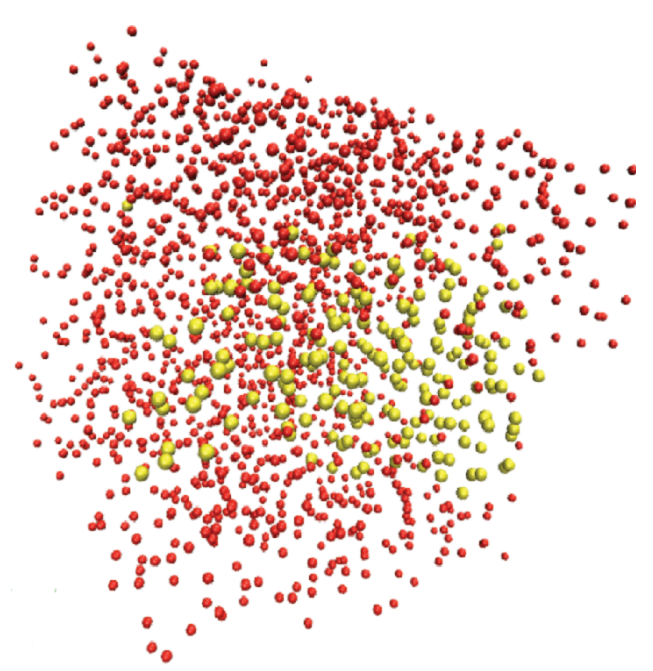

Fig. 4. (Color online) Same as the previous figure, but using the modified suboxide energies extracted from an "ideal" NC. 


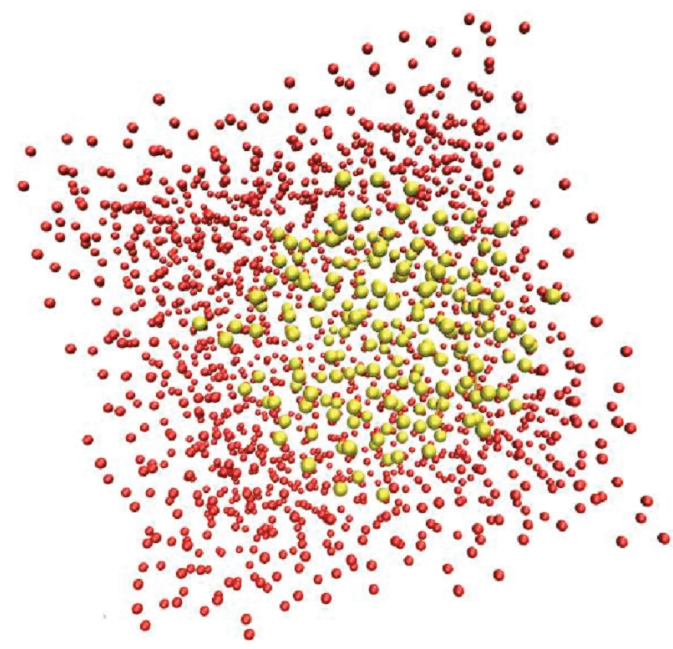

Fig. 5. (Color online) Same as the previous figure, but further invoking the asphericity and surface-to-volume constraints.

of asphericity parameter and the surface-to-volume ratio yields almost spherical NC as illustrated in Figure 5.

\subsection{Structural and Chemical Analysis}

The radial distribution function gives important knowledge about size and shape of the NC. In Figure 6 we present the radial distribution function of $\mathrm{Si}$ atoms with zero-oxidation state. First peak in this graph resembles mean bond length of $\mathrm{Si}-\mathrm{Si}$ bonds in the NC region while width of second peak resembles deviation of $\mathrm{Si}-\mathrm{Si}-\mathrm{Si}$ angles from diamond crystal state. Also radius and diameter of the NC can be read from Figure 6 since the probability of finding a pair with distance equal to radius is maximum and the probability of finding a pair within diameter distance is minimum in sphere-shaped NC. So from Figure 6 we can extract the radius of $\mathrm{NC}$ as: $\mathrm{r}_{N C}=11.3 \mathrm{~A}$. The radial distribution function of $\mathrm{Si}$ atoms with zero-oxidation state to four-oxidation state is presented in Figure 7. From this

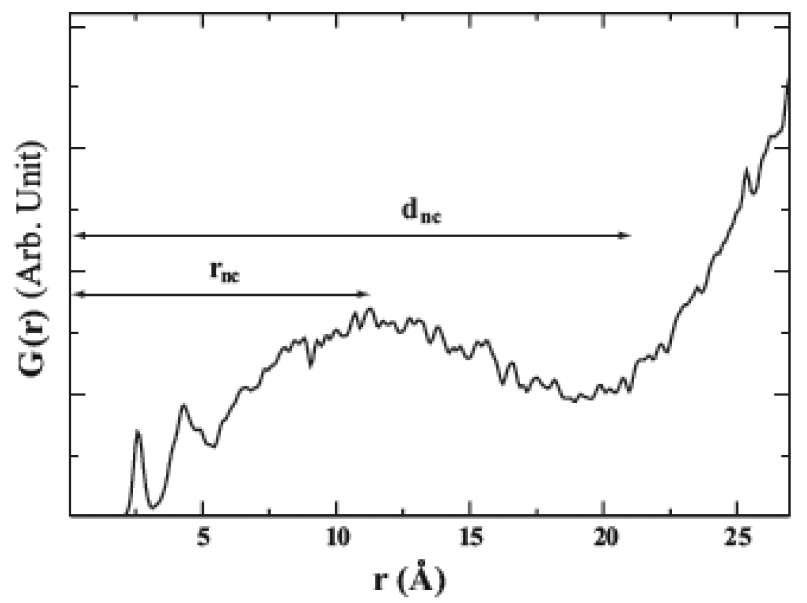

Fig. 6. Radial distribution function of zero-oxidation state $\mathrm{Si}$ atoms.

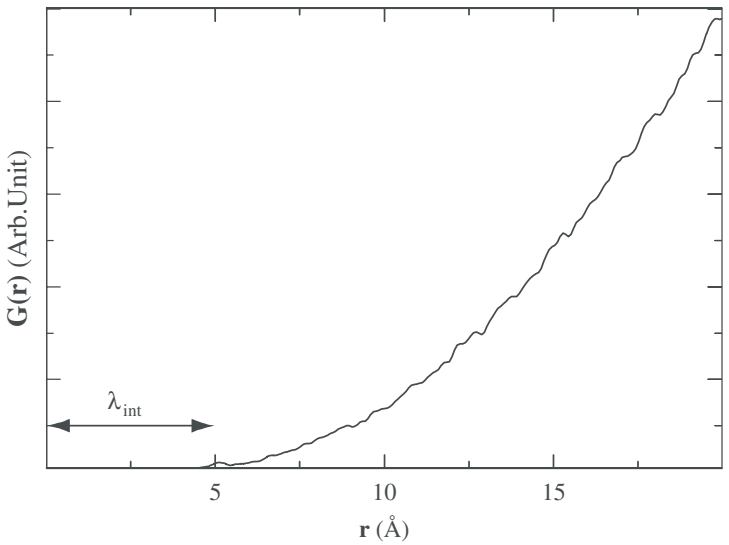

Fig. 7. Radial distribution function for the zero-oxidation state $\mathrm{Si}$ atoms to the four-oxidation state $\mathrm{Si}$ atoms.

graph, width of the interface region between $\mathrm{NC}$ and oxide can be read. Since there is no pair with distance less then $5.0 \AA$ we can conclude that width of the interface region is about 5.0 A. Once the interface regions are also accounted our predictions for the NC size as a function of oxygen molar fraction match reasonably well with the experimental data. ${ }^{4}$

Another valuable information can be extracted from the bond analysis across the interface of the NC. There is strong evidence that optical properties of the NCs are to some extend controlled by the interface region; especially the role of double ${ }^{11,12}$ and bridge bonds ${ }^{6}$ have been emphasized. Analysing the implicit bond topology of our final $\mathrm{NC}$, we observe that the fraction of $\mathrm{Si}-\mathrm{O}-\mathrm{Si}$ bridge bonds over all surface bonds is about $12 \%$. On the other hand, due to our Keating-type energetics, double bonds are not defined. This is one of the deficiencies of the current approach.

\section{CONCLUSIONS}

The atomistic NC structure simulation is attempted through oxygen diffusion process in amorphous siliconrich-oxide as governed by suboxide penalty energies. We observed that the available $a b$ initio suboxide energies are not satisfactory for this purpose, at least in this context, whereas one can extract them from "ideal" embedded NC suboxide densities. Even though this modification improves the sphericity of the NCs substantially, it still requires further shape constraints such as the minimization of the surface-to-volume ratio. The crystalline order, interface thickness and bond topology of the $\mathrm{NC}$ are quantitatively analyzed with this tool. The main drawback of the algorithm is the necessity for the shape constraints which breach the predictive power of the method. This study suggests the use of a more involved force field including longe-range forces as well as accommodating different chemical environments such as the double bonds. 
Acknowledgments: The authors would like to thank Dr. Can Uğur Ayfer for the access to Bilkent University Computer Center facilities. This work has been supported by the European FP6 Project SEMINANO with the contract number NMP4 CT2004 505285, by the Turkish Scientific and Technical Council TÜBITTAK with the project number 106T048 and BİDEB-2221 programme.

\section{References and Notes}

1. F. Wooten, K. Winer, and D. Weaire, Phys. Rev. Lett. 54, 1392 (1985).

2. P. N. Keating, Phys. Rev. 145, 637 (1966).
3. D. R. Hamann, Phys. Rev. B 61, 9899 (2000).

4. V. M. Burlakov, G. A. D. Briggs, A. P. Sutton, and A. Pasquarello, Phys. Rev. Lett. 93, 135501 (2004).

5. Y. Tu, J. Tersoff, and G. Grinstein, Phys. Rev. Lett. 81, 4899 (1998).

6. G. Hadjissavvas and P. C. Kelires, Phys. Rev. Lett. 93, 226104 (2004).

7. Y. Tu and J. Tersoff, Phys. Rev. Lett. 84, 4393 (2000).

8. K. Sato, T. Izumi, M. Iwase, Y. Show, H. Morisaki, T. Yaguchi, and T. Kamino, Appl. Surface Sci. 216, 376 (2003).

9. D. C. Rapaport, The Art of Molecular Dynamics Simulation, Cambridge University Press, Cambridge (1995).

10. G. Rudnick and G. Gaspari, J. Phys. A 4, L191 (1986).

11. A. Puzder, A. J. Williamson, J. C. Grossman, and G. Galli, Phys. Rev. Lett. 88, 097401 (2002).

12. M. Luppi and S. Ossicini, Mater. Sci. Eng. B 101, 34 (2003).

Received: 23 June 2006. Revised/Accepted: 10 November 2006. 